\title{
CONTENT CHARACTERIZATION USING WORD SHAPE TOKENS
}

\author{
Penelope Sibun and David S. Farrar \\ Fuji Xerox Palo Alto Laboratory, 3400 Hillview Avenue, Palo Alto, CA 94304 \\ sibun@pal.xerox.com, farrar@pal.xerox.com
}

\begin{abstract}
By quickly classifying character images into character shape categories, it is possible to altomatically extract syntactic information from the text of document images without optical character recognition. Using word shape rokens composed of these charater shapecodes, a proporly traincel text tagger can extract part-of -speceh information from scanned document images. Later components of al document processing system can then use this information to locate topics, characteri\%c document stylc, and assist in information retrieval.
\end{abstract}

\section{INTRODUCTION}

There arc many text processing tisks that we would like to accomplish, stch as document classification, text database structuring, matehing documents with queries, and topic characterization. The ficld of computational linguistics has developed a variety of techniques for accomplishing these tasks for text documents represented by character codes (c.g., ASClI). However, many documents for which we would like to use our atulomated techniques are not stored online in character-eoded format, but instead cxist only on paper. Optical character recognition (OCR) is a icchnique for converting scanned document images into character codes. By using OCR, document images can be converted into a form amenable to cxisting text processing techniques. I lowever, OCR is expensive, slow, and of ten inaccurate. Because of these drawbacks, we would like to avoid OCR if we can, or at the least, postpone using OCR until we are confident that a document wartants detailed processing. In other words, we would like a high-bandwidth document processing system that is sensitive cnough to detect desired doeument icaltures.

Our document understanding goals at the Fuji Xerox Palo Alto Laboratory include language determination (Nakayama and Spit\%, 1993; Sibun and Spit\%, forthcoming), content rharateriation, and style charaterization. Toward these goals, we are developing a set of methods for extracting inlormation from document images which do not depend on OCR. We have been working toward our goal of inexpensive content characterization by adapting a part-of-speech lagger to process word shape tokens rather than character coded words. Part-of-speceh tagging is a technique that has been developed and refined over the past several years, and it provides an incepensive, fast, and reliable source of information for recognizing noun phrases and other syntax-related text featutes which help chatacterize a decument's content.

In this paper, we describe how we combine our technology for determining word shape tokens with texttagging technology. We are developing systems that can extract noun phrases and other content characteristics using only word shape tokens that have been lagged with their parts of speceh. Using this approach, we can process document images quickly to determine whether OCR is warranted, for cxample, when a text is a likely match for keywords in a databass query.

In the next two sections, we describe how word shape tokens are derived; in section four, we discuss part-of specch lagging; in the following four sections, we describe in detail part-of-spech tagging using word shape tokens; in sections nine and ten we discuss our tesults.

\section{WORD SHAPE TOKEN CREATION}

In this section we brielly describe our system that constructs character shape codes and word shape tokens from a document image (for more detail, sec Nakayama and Spit\%, 1993; Sibun and Spit\%, fortheoming). Г० recognizc character shape codes from an image, some transformations are first made to correct for various scanning artifacts such as skew angle and text line curvature. On each text line, four horizontal lines define three significant yones: the areal between the baseline and the top of characters such as " $\mathrm{x}$ " is the $x$ zone; the arca above the $\mathrm{x}$-hoight level is the astender zone; the arca below the $x$-zone is the descender zone (figure 1). The text line is further divided into charartercells by verticial boundaries which delineate the connected components of each charater image.

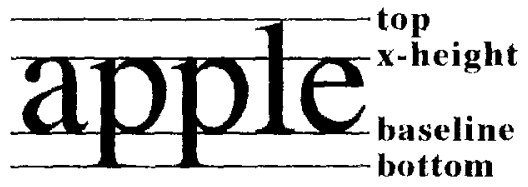

Figure 1: The text line parameter positions.

The majority of characters can easily be mapped to a small number of distinct codes (tigure 2). ${ }^{1}$ Characters which are containcd entircly in the $x$-zonc map to shape code $\mathbf{x}$; charaxeles which extend from the baseline to above the $\mathrm{X}$-height line map to shape code $\boldsymbol{\Lambda}$; and those which extend from below the baseline to the $\mathrm{x}$-height line map to shape code $\mathbf{g}$. Characters which map lo $\mathbf{A}, \mathbf{x}$, or $\mathbf{g}$ are composed of a single connected component. Some chatricters contain more than one connected component: an $x$-height character with a single diacritical mark in the ascender $/$ one maps to $\mathbf{i}$; a character with a descender and a single diacritical mark maps to j. Most common punctuation marks map to unique shape codes; however,

\footnotetext{
1 If this mapping can be done from document images, it call mote trivially be accomplished from character coded documents, such as ASC II text (providing, of course, that the method of encoding is known).
} 
some are mapped into shape codes shared with alphabelic characters (c.g., "\&" maps to shape code $\mathbf{A}$ ).

\begin{tabular}{|c|c|}
\hline Shape Coxde & Chatrater \\
\hline$\Lambda$ & $\Lambda . \%$ bdihk $110-9 / 7 \$ \& /(0)$ \\
\hline $\mathbf{x}$ & acem norsuraxy \\
\hline i & 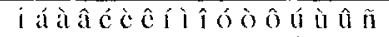 \\
\hline $\mathrm{g}$ & gp py \\
\hline $\mathbf{j}$ & $\mathrm{j}$ \\
\hline
\end{tabular}

Figure 2: Character shape codes.

\section{SHAPE CONVERSION}

In general, our approach to document processing finesses the problems inherent in mapping from an image to a chatracter-coded representation: we malp instead from the inage to a shape-based representation. 'This technique can transform even a degraded document image into a representation which provides usef ul abstractions about the text of al document. The shape-based representation that we construct is proving to be a remarkably rich source of information. While our initial goal hats been to use it lor language identification in support of downstream OCR processes, we are finding that this representation may be a sulficient source of intormation for document content chatacterization, such as that supported by part-of-speceh lagging.

In our lagging work, we have used character shape coded text derived from normal character-coled text. This is simply because we do not have aceess to cnough image documents on which to train a lagger. Wo call the process of creating a shape-based version of the document from the character conc based version shape conversion.

for the purpose of text lagging, then, we can think of the word shape token representation as an approximation of the representation composed of words. We can think about the relationship between words and word shape tokens as at matpping from a word to its corresponding word shape token. For example, the word "apple" maps to the word shape token $\mathbf{x g} \mathbf{g} \mathbf{\Lambda} \mathbf{x}$, and the word "apples" maps to the word shape token $\times \mathbf{g g} \mathbf{A} \times \mathbf{x}$.

In documents, words cxist as surjace forms, not as morphological systems; thus "apple" and "apples" are different words. Therefore, it is of no use to us to have al fexicon organized in terms of stcms and suffixes; instcad, our lexicon is composed of surface forms like "apple" and "apples". 'Throughout the rest of this paper, when we say "words", we mean words as surface lorms.

\section{PAR'T-OF-SPEECII TAGGING}

A part of speech lagger is a system that uses contcxt to assign parts of specch to words. Part-of-specch information facilitates higher-level analysis, such as recognizing noun phrases and other patterns in text. Several different approaches have been used for building text taggers. A particular form of Markov model has been widely used that assumes that a word depends probabilistically on just its part-ol-speceh calcerory, which in turn depends solely on the calcgories of the preceding two words. Fraining the model is sometimes done by means of a large tagged corpus, but this is not necessary.
The Baum-Welch algorithm (Baum, 1972), also known as the lorwatd-Backward algorithm, can be used. In this case, the model is called a hidden Markov model (IIMM), since state transitions (i.e., part-ol-spech categories) are assuncel to be unobservatic.

for this work, we usc an IMMM-based text lagger that is publicly arailable from Xerox PARC. As described in Culting et al. (1992), the PARC tagger is efficient and highly flexible. It is particularly important that the lagger can be trained on any corpus ol text, using any lexicon. This flexibility allows us to shape-comvert our training corpus and lexicon, as deseribed in section 5, without necling to modify the tagger itsell. Below we outline the basic operation of the PARC: lagger; please refer to Culting et al. (1992) for lurther detail.

1. Text destined for the lagger lirst encounters a tokenizer, whose duty is to convert text (at sequence of characters) inte a sequence of tokens. Fach sentenec boundary is also identilied by the tokentzer, and is passed as a special token.

2. The tokenizer passes tokens to the lexicon, where tokens are matched with a set of surface forms, cach annotated with a part-of-spech tag. 'The set of tags constitutes an ambiguity class. The lexicon passes along a strcam of (surface form, ambiguity class) pairs.

3a. In taining mode, the lagger lakes long sequences of ambiguity classes as input. It uses the Batun-Welch algorithm to produce a trained IMM, which is used as inpul in lagging mode. Training is performed on some corpus of interest; this corpus may be of broad coverage or maly be genre specific.

3b. In lagging mode, the tagger bufters sequenes of ambiguity classes between sentence boundaries. These sequences are disambiguated by computing the maximal path through the IIMM with the Viterbi algorithm (1967). Operating at sentence granularity does not sacrilice accuracy, since sentence boundarics are unambiguous. Oulput consists of pairs of surface forms and lags.

\section{THE ILXICON}

The word shape tagging in our work follows the IMM-based process described above. Both word shape lagging and standard word tagging requirc a lexicon.

\subsection{Constructing the Lexicon}

$\Lambda$ word shape lexicon can be derived from a standard lexicon of words. The lexicon used with the standard text tagger contains a list of all the distinct surface forms likely to be cncountered in the language. Associated with cach surlace form is a list of the possible parts of speceh that the sufface lorm can have. lor example:

$\begin{array}{ll}\text { atpple } & \text { noun } \\ \text { apples } & \text { plural noun } \\ \text { cat } & \text { verb } \\ \text { cats } & \text { third person singular verb } \\ \underline{\text { rod }} & \text { noun, adjective } \\ \text { the } & \text { detcrniner }\end{array}$

Once we have a lexicon which consists of surlace forms, we can use it to create al lexicon of word shape tokens for 
word shape tagging. In particular, this transformat, in consists of the following steps:

1. Shape convert the surface forms to thrit corresponding word shape tokens.

2. Sort the lexicon by surface form word shape. At this stage there may be duplicate word shape tokens.

3. Eliminate duplicate entries in the lexicon: collect all parts of speech behind one word shape token (combine their ambiguity classes). At this stage each word shape token should be unique.

4. Eliminate duplicate parts of specch behind each word shape token. At this stage each part of speceh should be unique within each ambiguity class.

The lexicon fragment above would be converted to:

$\mathbf{x g} \mathbf{g A x}$ noun

$\mathbf{x} \operatorname{ggAxx}$ plural noun

$\mathbf{x x A} \quad$ verb, noun, adjective

$\mathbf{x} \times \mathbf{A x} \quad$ third person singular verb

$\mathbf{A} \mathbf{A} \mathbf{x}$ determiner

\subsection{Analysis of the Lexicon}

For this work, we use a lexicon provided by Xcrox PARC. This lexicon is organized so that there is an entry for each of roughly 150,000 surface forms. lior word shape tagging, we shape converted this lexicon. $\Lambda$ s can be seen in the lable, shape conversion results in about 50,000 distinct word shape surface forms. This suggests that, on average, each word shape token is a mapping of threc surlace forms. However, about 30,000 of the word shape tokens are unique, that is, correspond to a single surfice form.

\begin{tabular}{|l|r|r|}
\cline { 2 - 3 } \multicolumn{1}{c|}{ Surface Forms } & Count & \%Total \\
\hline Standard Lexicon & 148,703 & 100.0 \\
\hline Shape-converted Lexicon & 47,102 & 31.7 \\
\hline Shape-converted Lnique & 28,949 & 19.5 \\
\hline
\end{tabular}

Thus, the word shape lexicon is approximatcly oncthird the size of the standard lexicon. Clearly, information has been lost, but not as much as one might think. In fact, the $20 \%$ of the word shape tokens that are unique carry cxactly as much information as their corresponding character-coded words. While some surface forms that map to unique word shape tokens are long and infrequent (like "llibbertigibbet", AAi AAxxAigiAAxA), many are short, common words:

\begin{tabular}{|c|c|}
\hline apple & $\operatorname{xgg} A x$ \\
\hline apples & $x \operatorname{ggA} \mathbf{A} x$ \\
\hline high & $\boldsymbol{A A} \mathbf{i g A}$ \\
\hline thirsty & $\mathbf{A} \mathbf{A} \times \mathbf{A} \mathrm{g}$ \\
\hline$\underline{\text { lifclike }}$ & $\operatorname{AiAx} \mathbf{A} \mathbf{i} \mathbf{x}$ \\
\hline galaxy & $\mathbf{g} \times \mathbf{A} \times \mathbf{g}$ \\
\hline payda & $g \times g \wedge \times g$ \\
\hline 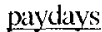 & $\operatorname{gxg} A \times g x$ \\
\hline
\end{tabular}

While word shape tokens that are unique have the same parts of speech as their corresponding surface forms, the others will tend on average to have many more parts of spech than an average surface form. This depends somewhat on the tagset (sec section 6). In general, word shape tokens frequently have as many as 10 to 15 parts of spech, whereas standard surface forms rarely have more than 4 or 5 .

\section{DEVISING THE TAGSET}

The lagset is implicit in the Icxicon: it includes all parts of specch listed in any cntry of the lexicon; it also includes a small set of tags for punctuation, stch as comma, hyphen, and sentence boundary. Nlthough the lagset is not explicitly defined, we can modily it by mapping from selected latgs found in the lexicon to other tags of our choosing. For cxample, the texicon distinguishes between verb tenses and has separate tags for different combinations of verb tensc, person, and number: present tense verb, past tense verb, third person singular present verb, cte. If we preferred, we could map all these different verb forms to at single veri tag. Itowever, we typically prefer to maintain such distinctions, as the text tagger can take advantage of differences in the surface forms of verbs with diflerent tenses in order to uniquely identify their parts of speech.

Shape conversion collapses different surlace lorms onto one word shape and merges their ambiguity classes. The result is that there tend to be fewer distinct surface lorms, and that cach surface form has, on average, a larger ambiguity class. If this ambiguity is problematic, one way to reduec it may be to reduec the size of the lagset. For example, we may choose to have one undifierentiated verb lag rather than a set which differentiates tense, person, and number. With fewer possible parts of speceh to choose from, the HMM may find the part-ol'-speceh selection more constrained. This in turn may improve its accuracy at selecting one of the tags that are atrailable.

The uninteresting case, of course, is where every word shape has the same tag, that is, a lag set of one. This situation yields no useful syntactic information from the document. Since the use of word shape tokens does reduce the amount of information that is avalable to the tagger, it may reduce the number of different tags it can accurately assign. The proper size of the lagset becomes constrained on one hand by the amount of syntactic information we wish to extract (nore information with a latger tagset) and on the other by the size of the ambiguity classes of the word shape tokens (more ambiguity with a larger lagset). Its proper size is thus an empirical question. For our tests we used tagsets with approximately 30 parts of spech.

\section{THE TRAINING PROCHSS}

Just as the hidden Markov model for standard text tagging requires a large corpus of text to train on, the word shape HMM requires a large corpus of text that has been converted to word shape tokens. We used at least 3.5 megabytes of ASCI text for our standard text lagger's corpus; we then shape converted this text to create the corpus for the word shape agger. This corpus ensisted of a varicty of different writing styles (from colloguial to prolessional) and difliculty levels (from casual to endite) Examples include essiays by humorists, proposals for new government policies, and classic works of licrature. 


\section{THE TAGGING PROCLSS}

With the word shape lexicon in place and an adequately trained IMM, word shape tagging works just as standard text lagging does. In particular, word shape lagging consists of the following steps:

1. A strean of text is tokenized into a strean of word shape tokens segmented into sentences.

2. The shape-converted lexicon assigns an ambiguty class to each word shape token. The result is a stream of sentencer compesed of (word shape token, ambiguity reass) pairs.

3. The tagger uses the trained hidden Markoy model to compute the highest probability part of specech for each word shape token in a sentence. The result is a stieam of (word shape token, part of speerh) pairs, which aro grouped according to sentence boundaries.

We can now use the resulting parts of speech to inlorm other segments of a document understanding system. Tho word shape part-ol-speech tagger thus aceepts word shape tokens grouped by sentence boundaries; within those boundaries, it assignts the most likely part of spech to each word shape totien.

\section{RESULTS}

In this section, we introfuce a tool which can recognize noun phrases in sentences, and we use this tool to compate the performance of the standard lagger and the word shape lagger. Wo exemplify the comparison with two lexts: one on which the standard lagger performs very well, and one en which it does relatively poorly. While the word shape tagger does less well in each case, its behavior tracks that of the standard tagger, exhibiting similar suecesses ated failures. I or the particular lask of finding simple noun phrases, the word shape lagger's performance is less than that of the standard lagger's, but a large faction of the noun plarases still are found.

We have a system that can recognize simple noun phrases when given as input the sequence of lags for a sentence. thach of these phases comprises a contiguous sequence of tags that satislies a simple grammar. for example, a noun phase can be simply a pronoun laye or an abitraty sequence of noun and adjective lags, possibly preceded by a determincr lage and possibly with an cmbedded possessive lag. 2 The longest possible such secpuences atre found. Conjanctions are not recognized as part of a noun phrase, nor is prepositional phrase allactment performod. We can be conlident of linding many simple noun phatses because the word "the" has tho unique word shipe A A $\mathbf{x} .{ }^{3}$ Recogntion of noun phrases is a liest step in topic identilication: the lopic of a document is likely to be indicated by its most frequent noun phrases.

In cvaluating the lagger eror tate, we use several measures (sec tables). We calculate the perentage of total crrors, the percentage of trivial errors, and the pereentags

2. The possessive tag is used for "s " or " 1 " as in "the call's pajamas' stripes"

3 Another linglish word, "lin," also mapes to $\mathbf{\Lambda} \mathbf{A} \mathbf{x}$ : fortutately, in most contexts this word is rale. of pernicionserrors (there are a lew crrots that do not fall in cither of the latter catcgories). 'Tagging "alarming" in "what the advocates are finding alarming" as a present participle rather than as an adjective is an example of a trivial enor. Pernicious errors typically involve mistagging nouns as verbs or verbs as nouns (in linglish, there are many surface forms that can be cither nominal of verbal). These latter errors cause problems in later processing, such as delecting simple noun phrases, since they maty obscurs noun phrases or introduce spurious ones.

We compare the standard lagger and the word shape lagger by counting the matches in the streams of output lags. Wo do not demand strict matches, but instead allow the lags to belong to pertinent equivalence classes. lior cxample, the standard tagger labels the noun "monitors" as a plural noun, and the word shape lagger labels $x \times \times$ i A $\times x \times$ simply as a noun. Wc consider this a match, since a noun and a plual noun are equally well recognived as part of a noun phrase.

Almost all instances of mismatches result from the standard laggel being right and the word slape tagger being wrong. Very occasionally the situation is the reverse, but this is to be expected as within the nommal range of probabilities. More interesting is the obscrvation that almost every pernicious crror made by the standard tagger is repeated by the word shape tagger. We take this as confirmation of the word shape lagger's ability to approximate the slandard lagger's pertormanee.

The first comparison of lagger performanec involves a 394-word excerpt from a government document. The standard lagger's performance is better than $95 \%$ correct, or better than $97 \%$ if trivial errors are distegarded. The word shape tagger's performance is a $59 \%$ match of the standard tagger's (or 5l\% if only exact matches are considered). The noun phrase recognizer lound 11.3 simple noun phrases in the standat dagger's output and $77(68 \%)$ of these in the word silape tagger's output.

\section{Standard Tagger Enorss}

\begin{tabular}{|c|c|c|c|c|}
\hline [exl & Totall & Trivial & Pernicious & ()this \\
\hline & $4.66^{\prime}$ & 4 & 2.3 & 0.9 \\
\hline Jonsel & $11.1 \%$ & $4.2 \%$ & $4.9 \%$ & \\
\hline
\end{tabular}

\section{Matching Output of}

Standard Tagger and Word Shape Tagger

\begin{tabular}{|c|c|c|}
\hline lexl & $\begin{array}{r}\text { Disrogatcing } \\
\text { Trivial Mismatches }\end{array}$ & $\begin{array}{l}\text { Including all } \\
\text { Mismatches }\end{array}$ \\
\hline Govel & $59 \%$ & $51 \%$ \\
\hline Nonscuse & $47 \%$ & $38 \%$ \\
\hline
\end{tabular}

Noull Pharases Recognized from 'lagger Output

\begin{tabular}{|l|r|r|}
\hline Texl & Standard & Word Shape \\
\hline Gowernment & 113 & 77 \\
\hline Nonsense & 45 & 17 \\
\hline
\end{tabular}

The second comparison is of lagging a 1/4-word pice of nonsense verse. 'The standard tagger's perfomanee is 
$89 \%$ correct, or $94 \%$ disregarding trivial crrors. The word shape tagger's performanec is a $47 \%$ mateh (or $38 \%$ considering only exact matches). The noun phrase recognizer found 45 simple noun phrases in the standard tagger's output and $17(38 \%)$ of these in the word shape lagger's output.

Further study is necded to determine exactly how reliable word shape part-of-speech lagging and simple noun phrase recognition will be in linding the topic or topies in a document image. One means of improving this reliability may be our technique for grammatical function assignment which uses only the output of the part-of-specch tagger and phrase recognizers (Sibun 1991). However, we can alteady use part-of-speceh tagging and simple noun phrase recognition as a tool for discerning somelhing about the content of the document by discovering at least some of its noun phrases. Since our document recognition technology allows us to use word shape tokens to index directly into the doeument image, we can also identily parts of the image as promising candidates for $O C R$.

\section{DISCUSSION}

Although the word shape tagger deals with greater ambiguity, it can still cxtract significant information from a text. The increase in ambiguity is not as high as might be expected: a large number of word shapes remain unambiguous after the lexicon has been shape eonverted. As noted above, the ereation of the word shape lexicon from the standard lexicon reduces the number of distinct entrics to approximately one-third. For example, distinct words such as "cat" and "rat" map onto the same word shape token $\times \mathbf{x} \mathbf{A}$. Nevertheless, the complexity of English spelling still allows a large proportion of surface forms to be distinguished merely by their word shapes.

Several improvements on our technique remain to be fully implemented. We do not yet have a principled way to detcrmine the optimal tagsel for a given corpus of text. As noted above, thete is a tension between the size of the tagset and the amount of syntactic information that is available in the word shape lokens.

We are also investigating computationally inexpensive ways of making finer distinctions between characters that map to the character shape codes $\mathbf{x}$ and $\mathbf{A}$. Initially, parentheses and brackets were always classified as $\mathbf{A}$ and distorted any word shape they were adjacent to: for cxample, "(USA)" would be shape converted to A A A A A. Recently we have made progress in recogniring these nonalphabetic characters ats word shape token delimiters, rather than parts of the word shape tokens themselves. It may also be useful to distinguish inore alphabetic character classes by mapping scanned character images to a larger set of character shape codes. We can extract more useful information by distinguishing upper case letters from lower ease letters, such as " $h$ " and " $k$ ", which map to the character shape code $\mathbf{A}$. A larger number of chatacter shape codes gives us more information about the word shape tokens, and helps to reduce ambiguity. However, we must be careful to choose character shape features which can be casily detected in the image and quickly classified by a character shape code.

In kecping with Fuji Xerox's multi-lingual document cmphasis, we are atso cxploring ways in which this method may be applice to other Roman-alphabet languages, such as French, German, Dutch, and Spanish. 'The techmique will need to be cvaluated separately for each language, however, to better understand how each langulage's typographic conventions may be reflected in its word shape.

\section{CONCLUSION}

We have presented a new technique for the understanding of English document images without optical character rcognition. By scanning and categorizing character shapes, it is possible to extract word shapes from the document text; these word shapes tokens can then be used as input to a lagger which determines part-of-speceh information. This part-of-spech information can then be used to inform other document understanding techniques, including noun phrase recognition and topic identification. The lack of OCR means we cannot extract all of the information contained in the scanned document's image; nevertheless, the information from the word shape tokens allows us to characterize the document's content with significant accuracy, and morc quickly than if we hakd performed OCR.

\section{Acknowledgments}

We thank Larry Spic and Masa Ozaki for their uselul comments.

\section{References}

Baum, L. E. "An incquality and associated maximization technique in statistical estimation for probabilistic functions of a Markov process." Inequalities, 3:1--8, 1972.

Culting, Doug, Julian Kupiec, Jan Pedersen, and Penclope Sibun. "A Practical Part-of-Speceh Tagger." In Procedings of the Third Conference on Applied Natural language Processing (ACL), pp 133-140), Trento, Italy, 1992. Also Repori SSL-92-01/P92(O)O()1, Xerox Palo Alto Research Center, 1992.

Nakayama, Takchiro and A. L. Spitr. "Europcan Language Determination from Image." In Proceedings of the Second International Conferenec on Document Analysis and Recognition, pp 159-162, Tsukuba Scicnec City, Japan, 1993.

Sibun, Penelope. "Grammatical Function Assignment in Unrestricted Text." Internal Report, Xcrox Palo Alto Research Center, 1991.

Sibun, Penclope and A. Lawrence Spitz. "Language Determination: Natural Language Processing from Scanned Document Images." Forthcoming.

Viterbi, A. J. "Error bounds for convolution codes and an asymplotically optimal decoling algorithm." IIIE Transactions on Information Theory. pp 260-269. April 1967. 